\title{
AN ISOPERIMETRIC INEQUALITY FOR LAPLACE EIGENVALUES ON THE SPHERE
}

\author{
MIKHAIL KARPUKHIN ${ }^{1}$, NIKOLAI NADIRASHVILI, ALEXEI V. PENSKOI ${ }^{2}$, \\ AND IOSIF POLTEROVICH ${ }^{3}$
}

\begin{abstract}
We show that for any positive integer $k$, the $k$-th nonzero eigenvalue of the Laplace-Beltrami operator on the two-dimensional sphere endowed with a Riemannian metric of unit area, is maximized in the limit by a sequence of metrics converging to a union of $k$ touching identical round spheres. This proves a conjecture posed by the second author in 2002 and yields a sharp isoperimetric inequality for all nonzero eigenvalues of the Laplacian on a sphere. Earlier, the result was known only for $k=1$ (J. Hersch, 1970), $k=2$ (N. Nadirashvili, 2002; R. Petrides, 2014) and $k=3$ (N. Nadirashvili and Y. Sire, 2017). In particular, we argue that for any $k \geqslant 2$, the supremum of the $k$-th nonzero eigenvalue on a sphere of unit area is not attained in the class of Riemannian metrics which are smooth outsitde a finite set of conical singularities. The proof uses certain properties of harmonic maps between spheres, the key new ingredient being a bound on the harmonic degree of a harmonic map into a sphere obtained by N. Ejiri.
\end{abstract}

\section{INTRODUCTION AND MAIN RESULTS}

Let $M$ be a closed surface and $g$ be a Riemannian metric on $M$. Consider the Laplace-Beltrami operator $\Delta: C^{\infty}(M) \longrightarrow C^{\infty}(M)$ associated with the metric $g$,

$$
\Delta f=-\frac{1}{\sqrt{|g|}} \frac{\partial}{\partial x^{i}}\left(\sqrt{|g|} g^{i j} \frac{\partial f}{\partial x^{j}}\right),
$$

and its eigenvalues

$$
0=\lambda_{0}(M, g)<\lambda_{1}(M, g) \leqslant \lambda_{2}(M, g) \leqslant \lambda_{3}(M, g) \leqslant \ldots,
$$

where each eigenvalue is repeated according to its multiplicity.

Let $\operatorname{Area}(M, g)$ denote the area of $M$ with respect to the Riemannian metric $g$. Since the eigenvalues possess a rescaling property,

$$
\forall t>0 \quad \lambda_{i}(M, t g)=\frac{\lambda_{i}(M, g)}{t}
$$

\footnotetext{
${ }^{1}$ Partially supported by Tomlinson Fellowship and Schulich Fellowship.

${ }^{2}$ Partially supported by the Simons-IUM Fellowship and by the Young Russian Mathematics award.

${ }^{3}$ Partially supported by NSERC, FRQNT and the Canada Research Chairs program.

2010 Mathematics Subject Classification. 58J50, 58E11, 53C42.
} 
it is natural to consider functionals

$$
\bar{\lambda}_{i}(M, g)=\lambda_{i}(M, g) \operatorname{Area}(M, g),
$$

invariant under the rescaling transformation $g \mapsto t g$. The functional $\bar{\lambda}_{i}(M, g)$ is sometimes called an eigenvalue normalized by the area or simply a normalized eigenvalue.

Our principal interest is in the following geometric optimization problem for the Laplace-Beltrami eigenvalues: given a compact surface $M$ and a number $i \in \mathbb{N}$ find the quantity

$$
\Lambda_{i}(M)=\sup _{g} \bar{\lambda}_{i}(M, g),
$$

where the supremum is taken over the space of all Riemannian metrics $g$ on M.

Since the functional $\bar{\lambda}_{i}(M, g)$ is invariant under rescaling of the metric $g \mapsto t g$, where $t \in \mathbb{R}_{+}$, this problem is equivalent to the question of finding $\sup \lambda_{i}(M, g)$, where the supremum is taken over the space of all Riemannian metrics $g$ of area 1 on $M$. This problem is often referred to as the isoperimetric problem for the Laplace-Beltrami eigenvalues, since for any metric $g$ of unit area on $M$, one has the inequality

$$
\lambda_{i}(M, g) \leqslant \Lambda_{i}(M) .
$$

Definition 1.1. Let $M$ be a closed surface. A metric $g_{0}$ on $M$ is called maximal for the functional $\bar{\lambda}_{i}(M, g)$ if

$$
\Lambda_{i}(M)=\bar{\lambda}_{i}\left(M, g_{0}\right)
$$

Note that if a maximal metric exists, it is defined up to multiplication by a positive constant due to the rescaling invariance of the functional.

The main goal of the present paper is to provide a complete solution of the isoperimetric problem for the Laplace-Beltrami eigenvalues on a sphere. We will say that a metric on a sphere is round if it is isometric to the standard round metric of constant Gaußian curvature. We will also say that the two round spheres are touching if they have exactly one point in common. In what follows, the choice of the points where the touching occurs is of no significance, as it does not change the spectrum (note the spectra of touching and disjoint spheres are the same).

Theorem 1.2. Let $\left(\mathbb{S}^{2}, g\right)$ be the sphere endowed with a metric $g$ which is smooth outside a finite number of conical singularities. Then the following inequality holds:

$$
\bar{\lambda}_{k}\left(\mathbb{S}^{2}, g\right) \leqslant 8 \pi k, \quad k \geqslant 1 .
$$

For $k=1$, the equality is attained if and only if $g$ is a round metric. For $k \geqslant 2$ the inequality is strict, and the equality is attained in the limit by a sequence of metrics degenerating to a union of $k$ touching identical round spheres. 
Theorem 1.2 immediately implies

$$
\Lambda_{k}\left(\mathbb{S}^{2}\right)=8 \pi k, \quad k \geqslant 1 .
$$

It settles a conjecture that was proposed in 2002 by the second author [40] (see also [44, as well as [26] for numerical evidence supporting the conjecture). For $k=1$, the result is a classical theorem due to J. Hersch [23]. For $k=2$, the proof of the equality (4) was outlined in [40, and a different argument was found later in [52]. Recently, (44) has been also established in the case $k=3$ [44]. For $k \geqslant 4$, Theorem 1.2 is new.

Remark 1.3. The degenerating sequence of metrics in Theorem 1.2 illustrates the "bubbling phenomenon" arising in the maximization of higher eigenvalues, see 43 for details. It would be interesting to check whether the equality in (3) could be attained in the limit only by a sequence of metrics converging to a union of touching spheres, or there exist other maximizing sequences.

Remark 1.4. It is instructive to compare Theorem 1.2 with isoperimetric inequalities for other eigenvalue problems. In particular, one could draw an analogy with the Hersch-Payne-Schiffer inequality for the $k$-th nonzero Steklov eigenvalue on simply connected planar domains, which is sharp for all $k \geqslant 1$, and the equality is attained in the limit as the domain degenerates into a disjoint union of $k$ identical disks [15]. The situation is quite different for higher Dirichlet and Neumann eigenvalues on planar domains. In this case, sharp isoperimetric inequalities for eigenvalues for $k \geqslant 3$ are not known, and numerical experiments indicate that optimal shapes may have rather complicated geometries [1. Moreover, the celebrated Polya's conjecture (see $[54,55,36]$ ) suggests that the $k$-th Dirichlet (respectively, nonzero Neumann) eigenvalue is bounded below (respectively, above) for all $k \geqslant 1$ by the main term in Weyl's asymptotics, which is not the case for inequality (3) (see 14, Remark 1.2.8] for a further discussion).

Organization of the paper. In the next section we give a brief overview of results on isoperimetric inequalities and extremal metrics for LaplaceBeltrami eigenvalues on surfaces. In Section 3 we recall the relation between extremal metrics, minimal immersions into spheres and harmonic maps. We also extend our considerations to harmonic immersions with branch points and metrics with conical singularities. In Section 4 we state the CalabiBarbosa theorem on harmonic immersions $\mathbb{S}^{2} \longrightarrow \mathbb{S}^{n}$ with branch points, and define the notion of the harmonic degree. Up to this point, our exposition follows closely [41] where similar ideas have been used to obtain a sharp isoperimetric inequality for the second eigenvalue on the projective plane. The main novel ingredient of our approach is introduced at the end of Section 4. It is an upper bound due to N. Ejiri [1] on the harmonic degree of a harmonic immersion $f: \mathbb{S}^{2} \longrightarrow \mathbb{S}^{2 m}$ in terms of the index $k$ of the eigenvalue $\lambda_{k}$ extremized by the metric induced by $f$. The proof of Theorem 1.2 is presented in Section [5. For the convenience of the reader, 
in Appendix $\mathrm{A}$ we sketch the proof of Ejiri's bound, which uses techniques from algebraic geometry. These methods are relatively new in spectral geometry and appear to be significant in the study of extremal metrics for Laplace-Beltrami eigenvalues (see also [46]).

Acknowledgements. A.P. is very grateful to the Institut de Mathématiques de Marseille (I2M, UMR 7373) for its hospitality. The authors would like to thank G. Kokarev, E. Lauret and the anonymous referees for helpful remarks.

\section{ISOPERIMETRIC INEQUALITIES AND EXTREMAL METRICS FOR EIGENVALUES ON SURFACES}

In this section we present a brief overview of the main developments in the study of the isoperimetric inequalities and extremal metrics for the eigenvalues of the Laplace-Beltrami operator on surfaces (see also [56, section III.8]). The story began almost half a century ago when Hersch [23] obtained a sharp isoperimetric inequality for the first eigenvalue on a sphere which was mentioned in the previous section. Later, Yang and Yau [59] proved that if $M$ is an orientable surface of genus $\gamma$ then

$$
\bar{\lambda}_{1}(M, g) \leqslant 8 \pi(\gamma+1) .
$$

Actually, the arguments of Yang and Yau imply a stronger, though in general still non-sharp estimate,

$$
\bar{\lambda}_{1}(M, g) \leqslant 8 \pi\left[\frac{\gamma+3}{2}\right],
$$

see [16]. Here [.] denotes the integer part of a number.

A similar result in the non-orientable case has been recently obtained in [30], see also [32]. For a non-orientable surface $M$ of genus $\gamma$,

$$
\bar{\lambda}_{1}(M, g) \leqslant 16 \pi\left[\frac{\gamma+3}{2}\right] .
$$

Here the genus of a non-orientable surface is defined to be the genus of its orientable double covering.

For higher eigenvalues, Hassannezhad [22, building on the earlier work of Korevaar [33, proved that there exists a constant $C$ such that for any $i>0$ and any compact surface $M$ of genus $\gamma$, the following upper bound holds:

$$
\bar{\lambda}_{i}(M, g) \leqslant C(\gamma+i) .
$$

These results imply that the functionals $\bar{\lambda}_{i}(M, g)$ are bounded from above, and, as a result, $\Lambda_{i}(M)<+\infty$. A number of important results on the existence and regularity of maximizing metrics has been recently obtained, see [31, 42, 43, 51]. However, the list of known values $\Lambda_{i}(M)$ for all surfaces other than the sphere is quite short. 
$\mathrm{Li}$ and Yau proved in 1982 in the paper 35 that the standard metric on the projective plane is the unique maximal metric for $\bar{\lambda}_{1}\left(\mathbb{R} \mathbb{P}^{2}, g\right)$ and

$$
\Lambda_{1}\left(\mathbb{R P}^{2}\right)=12 \pi \text {. }
$$

The second author proved in 1996 in the paper [39] that the standard metric on the equilateral torus is the unique maximal metric for $\bar{\lambda}_{1}\left(\mathbb{T}^{2}, g\right)$ and

$$
\Lambda_{1}\left(\mathbb{T}^{2}\right)=\frac{8 \pi^{2}}{\sqrt{3}}
$$

Note that the functional $\bar{\lambda}_{i}(M, g)$ depends continuously on the metric $g$. However, when $\bar{\lambda}_{i}(M, g)$ is a multiple eigenvalue this functional is not in general differentiable. If we consider an analytic variation $g_{t}$ of the metric $g=g_{0}$, then it was proved by Berger [4, Bando and Urakawa [2], El Soufi and Ilias [19] that the left and right derivatives of the functional $\bar{\lambda}_{i}\left(M, g_{t}\right)$ with respect to $t$ exist. This leads us to the following definition given by the first author in the paper [39] and by El Soufi and Ilias in the papers [18, 19].

Definition 2.1. A Riemannian metric $g$ on a closed surface $M$ is called an extremal metric for the functional $\bar{\lambda}_{i}(M, g)$ (or simply for the eigenvalue $\lambda_{i}$ ) if for any analytic deformation $g_{t}$ such that $g_{0}=g$ one has

$$
\left.\left.\frac{d}{d t} \bar{\lambda}_{i}\left(M, g_{t}\right)\right|_{t=0+} \cdot \frac{d}{d t} \bar{\lambda}_{i}\left(M, g_{t}\right)\right|_{t=0-} \leqslant 0 .
$$

It was proved in [25] that the metric on the Klein bottle realized as the so-called bipolar Lawson surface $\tilde{\tau}_{3,1}$, is extremal for $\bar{\lambda}_{1}(\mathbb{K} \mathbb{L}, g)$. It was conjectured in this paper that this metric is the unique extremal metric and, moreover, the maximal one. It was later shown by El Soufi, Giacomini and Jazar [13] that this metric on $\tilde{\tau}_{3,1}$ is indeed the unique extremal metric for the functional $\bar{\lambda}_{1}(\mathbb{K} \mathbb{L}, g)$. Moreover, one can prove that $\Lambda_{1}(\mathbb{K} \mathbb{L})$ is attained on a smooth metric [8], and therefore the metric on $\tilde{\tau}_{3,1}$ is the maximal one. Hence,

$$
\Lambda_{1}(\mathbb{K} \mathbb{L})=\bar{\lambda}_{1}\left(\mathbb{K} \mathbb{L}, g_{\tilde{\tau}_{3,1}}\right)=12 \pi E\left(\frac{2 \sqrt{2}}{3}\right),
$$

where $E$ is the complete elliptic integral of the second kind and $g_{\tilde{\tau}_{3,1}}$ is the metric on $\tilde{\tau}_{3,1}$.

More results on extremal metrics on tori and Klein bottles could be found in the papers [18, 27, 28, 29, 34, 47, 48, 50]. A review of these results is given by the third author in the paper [49].

It was shown in 24] using a combination of analytic and numerical tools that the maximal metric for the first eigenvalue on the surface of genus two is the metric on the Bolza surface $\mathcal{P}$ induced from the canonical metric on the sphere using the standard covering $\mathcal{P} \longrightarrow \mathbb{S}^{2}$. The authors stated this result as a conjecture, because the argument is partly based on a numerical calculation. The proof of this conjecture was presented in a recent preprint [46]. 
For surfaces of genus $\gamma \geqslant 3$, no maximal metrics for Laplace eigenvalues are known. The only surface other than the sphere for which a sharp isoperimetric inequality was established for a higher eigenvalue is the projective plane. It was shown in [4] that

$$
\Lambda_{2}\left(\mathbb{R P}^{2}\right)=20 \pi \text {. }
$$

It turns out that there is no maximal metric, but the supremum could be obtained as a limit on a sequence of metrics converging to a union of a projective plane and a sphere touching at a point, each endowed with the standard metric, such that the ratio of the areas of the projective plane and the sphere is $3: 2$. Inspired by this result, as well as by Theorem 1.2, we propose the following conjecture.

Conjecture 2.2. The equality

$$
\Lambda_{k}\left(\mathbb{R} \mathbb{P}^{2}\right)=4 \pi(2 k+1)
$$

holds for any $k \geqslant 1$. For $k \geqslant 2$ the supremum can not be attained on a smooth metric, and is realized in the limit by a sequence of metrics degenerating to a union of $k-1$ identical round spheres and a standard projective plane touching each other, such that the ratio of the areas of the projective plane and the spheres is $3: 2$.

Let us conclude this section by a result which is a special case of [53, Theorem 2]; it could be also deduced from [43, Theorem 1.1]. We use here that there exists only one conformal structure on $\mathbb{S}^{2}$.

Theorem 2.3. Let $k \geqslant 2$ and suppose that the following strict inequality holds:

$$
\Lambda_{k}\left(\mathbb{S}^{2}\right)>\max _{\substack{1<s \leqslant k \\ k_{1}+\cdots+k_{s}=k \\ k_{1}, \ldots, k_{s} \geqslant 1}} \sum_{j=1}^{s} \Lambda_{k_{j}}\left(\mathbb{S}^{2}\right) .
$$

Then there exists a maximal metric $g$, which is smooth except possibly at a finite number of conical singularities, such that $\Lambda_{k}\left(\mathbb{S}^{2}\right)=\bar{\lambda}_{k}\left(\mathbb{S}^{2}, g\right)$.

Theorem 2.3 will be used in an essential way in the proof of Theorem 1.2 , see Section 5 ,

Remark 2.4. As mentioned in [53], if one replaces the strict inequality in (5) by a non-strict inequality, then by a gluing argument it is always true. Indeed, assume that the maximum $\mu_{k}$ of the right hand side is achieved for some $k_{1}, \ldots, k_{s}$. Take $s$ copies of the sphere, and on the $j$-th copy, $j=1, \ldots, s$, consider a metric $g_{j}$ such that $\bar{\lambda}_{k_{j}}\left(\mathbb{S}^{2}, g_{j}\right)$ is close to $\Lambda_{k_{j}}\left(\mathbb{S}^{2}\right)$. Scale $g_{j}$ by a positive constant $t_{j}$ such that $\lambda_{k_{j}}\left(\mathbb{S}^{2}, t_{j} g_{j}\right)$ is independent of $j$. Gluing these spheres together using sufficiently thin and short tubes yields a metric $g$ on the sphere with $\bar{\lambda}_{k}\left(\mathbb{S}^{2}, g\right)$ close to $\mu_{k}$ (see [9, Section 2] for details). Since $g_{j}$ could be chosen in such a way that $\bar{\lambda}_{k_{j}}\left(\mathbb{S}^{2}, g_{j}\right)$ is arbitrary 
close to $\Lambda_{k_{j}}\left(\mathbb{S}^{2}\right)$ for all $j=1, \ldots, s$, and the connecting tubes could be made arbitrary small, we get $\Lambda_{k}\left(\mathbb{S}^{2}\right) \geqslant \mu_{k}$ which proves the claim.

Remark 2.5. In fact, both [53, Theorem 2] and [43, Theorem 1.1] are concerned with the existence of Riemannian metrics with conical singularities for which the quantity

$$
\Lambda_{k}(M,[g])=\sup _{h \in[g]} \bar{\lambda}_{k}(M, h)
$$

is attained. The existence is guaranteed provided the condition [53, formula (0.3)] holds. Using Theorem 1.2 one can simplify this condition, see discussion in [53, page 5]:

Corollary 2.6. Let $(M, g)$ be a Riemannian surface and $k \geqslant 2$. If

$$
\Lambda_{k}(M,[g])>\Lambda_{k-1}(M,[g])+8 \pi,
$$

then there exists a maximal metric $\tilde{g} \in[g]$, smooth except possibly at a finite set of conical singularities, such that $\Lambda_{k}(M,[g])=\bar{\lambda}_{k}(M, \tilde{g})$.

\section{EXTREMAL METRICS, MiNIMAL IMMERSIONS AND HARMONIC MAPS}

Let us recall the definition of a minimal map, see e.g. [7, 10].

Definition 3.1. Let $M$ and $N$ be smooth manifolds and $h$ be a Riemannian metric on $N$. An immersion $f: M \rightarrow N$ is called minimal $f$ if it is extremal for the volume functional

$$
V[f]=\int_{M} d V o l_{f^{*} h}
$$

If $M$ is a Riemannian manifold endowed with a metric $g=f^{*} h$, then $f$ is called an isometric minimal immersion and $M$ is referred to as an (immersed) minimal submanifold.

It is well-known that a submanifold $M \rightarrow \mathbb{R}^{n}$ is minimal if and only if the coordinate functions $x^{i}$ are harmonic with respect to the LaplaceBeltrami operator on $M$. Since harmonic functions are eigenfunctions with eigenvalue 0 , it is natural to ask what is an analogue of this statement for a non-zero eigenvalue. The answer was given by Takahashi in 1966.

Theorem 3.2 (Takahashi [58]). If an isometric immersion

$$
f: M \leftrightarrow \mathbb{R}^{n+1}, \quad f=\left(f^{1}, \ldots, f^{n+1}\right),
$$

is defined by eigenfunctions $f^{i}$ of the Laplace-Beltrami operator $\Delta$ with a common eigenvalue $\lambda$,

$$
\Delta f^{i}=\lambda f^{i}
$$

then

(i) the image $f(M)$ lies on the sphere $\mathbb{S}_{R}^{n}$ of radius $R$ with the center at the origin such that

$$
\lambda=\frac{\operatorname{dim} M}{R^{2}}
$$


(ii) the immersion $f: M \leftrightarrow \mathbb{S}_{R}^{n}$ is minimal.

If

$$
f: M \leftrightarrow \mathbb{S}_{R}^{n} \subset \mathbb{R}^{n+1}, \quad f=\left(f^{1}, \ldots, f^{n+1}\right),
$$

is an isometric minimal immersion of a manifold $M$ into the sphere $\mathbb{S}_{R}^{n}$ of radius $R$, then $f^{i}$ are eigenfunctions of the Laplace-Beltrami operator $\Delta$,

$$
\Delta f^{i}=\lambda f^{i}
$$

with the same eigenvalue $\lambda$ given by formula (6) .

Let us introduce the counting function for the Laplace eigenvalues of a manifold $(M, g)$ :

$$
N(\lambda ; M, g)=\#\left\{\lambda_{i}(M, g)<\lambda\right\} .
$$

Theorem 3.2 implies that if $M$ is isometrically minimally immersed in the sphere $\mathbb{S}_{R}^{n}$, then the eigenvalue $\frac{\operatorname{dim} M}{R^{2}}$ is multiple. It is easy to see that $\lambda_{N\left(\frac{\operatorname{dim} M}{R^{2}} ; M, g\right)}(M, g)$ is the first Laplace eigenvalue equal to $\frac{\operatorname{dim} M}{R^{2}}$, where $g$ is the metric on $M$ induced by the immersion. This fact is important due to the following theorem.

Theorem 3.3 (Nadirashvili [39], El Soufi, Ilias [19]). Let $M \rightarrow \mathbb{S}_{R}^{n}$ be an immersed minimal compact submanifold. Then the metric $g$ induced on $M$ by this immersion is extremal for the eigenvalue $\lambda_{N\left(\frac{\operatorname{dim} M}{R^{2}} ; M, g\right)}$.

If a metric $g$ on a compact manifold $M$ is extremal for the $k$-th eigenvalue, then $g$ is induced by an isometric minimal immersion $M \rightarrow \mathbb{S}_{R}^{n}$ to the sphere of some dimension $n \geqslant 2$ and radius $R=\sqrt{\operatorname{dim} M / \lambda_{k}(M, g)}$. Moreover, the components of the immersion are given by the $\lambda_{k}$-eigenfunctions of the Laplacian.

We later use Theorem 3.3 for $M=\mathbb{S}^{2}$. In this case $\operatorname{dim} M=2$. Using rescaling one can consider only the case $R=1$. Hence, the value

$$
N\left(\frac{\operatorname{dim} \mathbb{S}^{2}}{R^{2}} ; \mathbb{S}^{2}, g\right)=N\left(2 ; \mathbb{S}^{2}, g\right)
$$

is particularly significant for our further considerations.

Let us now recall the definition of a harmonic map, see e.g. the review [7].

Definition 3.4. Let $(M, g)$ and $(N, h)$ be Riemannian manifolds. A smooth map $f: M \longrightarrow N$ is called harmonic if $f$ is extremal for the energy functional

$$
E[f]=\int_{M}|d f(x)|^{2} d V o l_{g}
$$

Here, $d f$ is viewed as a section of $T^{*} M \otimes f^{*} T N$ with the metric induced by $g$ and $h$. In local coordinates

$$
|d f|^{2}=g^{k l} \frac{\partial f^{i}}{\partial x^{k}} \frac{\partial f^{j}}{\partial x^{l}} h_{i j}
$$


The following theorem (see, e.g. the paper [10]) explains the relation between minimal and harmonic maps in the class of isometric immersions.

Theorem 3.5. Let $M, N$ be Riemannian manifolds. If $f: M \rightarrow N$ is an isometric immersion, then $f$ is harmonic if and only if $f$ is minimal.

It is well-known that if $\operatorname{dim} M=2$, then the property of $f$ to be harmonic depends only on the conformal class of the metric $g$, see e.g. 7]. A map $f:(M, g) \longrightarrow(N, h)$ is called conformal if the metrics $g$ and $f^{*} h$ belong to the same conformal class. Together with Theorem 3.5 this yields the following corollary.

Corollary 3.6. Let $(M, g)$ be a Riemannian surface. A conformal immersion $f:(M, g) \rightarrow(N, h)$ is harmonic iff $f: M \rightarrow(N, h)$ is minimal.

It is a well-known fact that there is only one conformal class of Riemannian metrics on $\mathbb{S}^{2}$. Hence, for $M=\mathbb{S}^{2}$ any harmonic immersion is conformal. Therefore, Theorem 3.3 implies:

Corollary 3.7. The smooth extremal metrics for the eigenvalues of the Laplace-Beltrami operator on the sphere $\mathbb{S}^{2}$ are exactly the metrics induced on $\mathbb{S}^{2}$ by harmonic immersions $f: \mathbb{S}^{2} \rightarrow \mathbb{S}^{n}$, where $\mathbb{S}^{n}$ is endowed with a round metric.

However, as one can see from Theorem 2.3 it is not sufficient to consider smooth metrics as one has to account for a possible presence of conical singularities. This is done by allowing minimal immersions to have branch points. According to [21], it is equivalent to replacing conformal harmonic maps with weakly conformal harmonic maps.

Definition 3.8. A map $f:(M, g) \rightarrow(N, h)$ is called weakly conformal if $f^{*} h=\alpha g$, where $\alpha \geqslant 0$ is a non-negative smooth function with isolated zeroes, i.e. the differential of $f$ can be degenerate at isolated points of $M$.

For a more detailed discussion on minimal immersions with branch points and metrics with conical singularities, see [8, 41]. In particular, Theorem 3.3 can be generalized to this context, see [31, Corollary 4.7]. In the special case $M=\mathbb{S}^{2}$ we have:

Theorem 3.9. Let $f: \mathbb{S}^{2} \rightarrow \mathbb{S}^{n}$ be a harmonic map. Then the metric $g$ (possibly with conical singularities) on $\mathbb{S}^{2}$ induced by this map is extremal for the eigenvalue $\lambda_{N\left(2 ; \mathbb{S}^{2}, g\right)}$.

If a metric $g$ (possibly with conical singularities) on $\mathbb{S}^{2}$ is extremal for the $k$-th eigenvalue, then $g$ coincides up to a rescaling with the metric induced by a harmonic map $\mathbb{S}^{2} \rightarrow \mathbb{S}^{n}$ to the unit sphere of some dimension $n \geqslant 2$. Moreover, $\lambda_{k}=2$ and the components of the harmonic map are given by the $\lambda_{k}$-eigenfunctions of the Laplacian.

Remark 3.10. As was confirmed to us by G. Kokarev, the "if" part of 31, Corollary 4.7] assumes additionally that $\lambda_{k-1}<\lambda_{k}$ or $\lambda_{k}<\lambda_{k+1}$, cf. [19, 
Theorem 3.1]. Note that by definition (17) of the counting function, $\lambda_{N\left(2 ; \mathbb{S}^{2}, g\right)}$ satisfies the former inequality, i.e. it is strictly greater than the preceding eigenvalue.

Let us also remark that for any manifold equipped with a metric with isolated conical singularities it is possible to construct a sequence of smooth Riemannian manifolds such that their area as well as their eigenvalues converge to the area and eigenvalues of the initial manifold, see e.g. [57.

\section{Harmonic maps into SPheres and the harmonic Degree}

We now focus on harmonic immersions $\mathbb{S}^{2} \uparrow \mathbb{S}^{n}$ with branch points. Let us introduce the following useful definition.

Definition 4.1. A harmonic map $f: \Sigma \longrightarrow \mathbb{S}^{n} \subset \mathbb{R}^{n+1}$ of a surface $\Sigma$ to the standard unit sphere $\mathbb{S}^{n} \subset \mathbb{R}^{n+1}$ is called linearly full if the image $f(\Sigma)$ does not lie in a hyperplane of $\mathbb{R}^{n+1}$.

The following theorem was proved by Calabi in 1967 and later refined by Barbosa in 1975. Let $g_{\mathbb{S}^{n}}$ denote the standard metric on $\mathbb{S}^{n}$ of radius 1 .

Theorem 4.2 (Calabi [6], Barbosa [3]). Let $f: \mathbb{S}^{2} \longrightarrow \mathbb{S}^{n}$ be a linearly full harmonic immersion with branch points. Then

(i) the area of $\mathbb{S}^{2}$ with respect to the induced metric Area $\left(\mathbb{S}^{2}, f^{*} g_{\mathbb{S}^{n}}\right)$ is an integer multiple of $4 \pi$;

(ii) $n$ is even, $n=2 m$, and

(iii)

$$
\operatorname{Area}\left(\mathbb{S}^{2}, f^{*} g_{\mathbb{S}^{n}}\right) \geqslant 2 \pi m(m+1) .
$$

Definition 4.3. If Area $\left(\mathbb{S}^{2}, f^{*} g_{\mathbb{S}^{n}}\right)=4 \pi d$, then we say that $f$ is of harmonic degree $d$.

Theorem 4.2 implies the following proposition.

Proposition 4.4. Extremal metrics (possibly with conical singularities) on $\mathbb{S}^{2}$ are induced by linearly full harmonic immersions with branch points $f: \mathbb{S}^{2} \longrightarrow \mathbb{S}^{2 m}$. The harmonic degree $d$ of such an immersion satisfies the inequality $d \geqslant \frac{m(m+1)}{2}$.

Indeed, any non-linearly full immersion can be a viewed as linearly full immersion to a corresponding subsphere.

As we know from Theorem 3.9, a metric $g$ induced by a linearly full harmonic immersion with branch points $f: \mathbb{S}^{2} \longrightarrow \mathbb{S}^{2 m}$ is extremal for the eigenvalue $\lambda_{N(2)}$, where

$$
N(2):=N\left(2 ; \mathbb{S}^{2}, g\right) .
$$

To simplify notation, we will use this convention in the remainder of the paper.

A priori, it is not clear how to find $N(2)$ for a metric on a surface induced by a harmonic immersion into a sphere. The following lower bound on $N(2)$ 
due to Ejiri is a particular case of [11, Theorem C] and is crucial for our considerations.

Theorem 4.5 (Ejiri [1]). Let $f: \mathbb{S}^{2} \longrightarrow \mathbb{S}^{2 m}$ be a linearly full harmonic map of harmonic degree $d>1$ of $\mathbb{S}^{2}$ to the standard unit sphere $\mathbb{S}^{2 m}$. Then

$$
N(2) \geqslant d+1 \text {. }
$$

We note that while the condition $d>1$ is not explicitly stated in the formulation of Theorem $\mathrm{C}$ in [11, it is assumed and used in its proof. For details, see Appendix A,

\section{Proof of Theorem 1.2}

As was mentioned in the introduction, it is known that $\Lambda_{k}\left(\mathbb{S}^{2}\right)=8 \pi k$ for $k=1([23]), k=2([40,52])$ and $k=3([44])$.

Let us first show that for $k=1$ a round metric is, up to rescaling, the unique maximal metric on the sphere among all metrics that are smooth outside a finite number of conical singularities. This is well-known for smooth metrics. Indeed, it was proved in 37. (see also [17]) that there exists at most one metric in a conformal class admitting an isometric immersion into a unit sphere by the first eigenfunctions. Given that there is only one conformal structure on $\mathbb{S}^{2}$, the uniqueness of the maximal metric readily follows from Theorem 3.3. It remains to show that a maximal metric for the first eigenvalue on the sphere is smooth. It follows from Proposition 4.4 that a maximal metric is induced by a linearly full harmonic immersion with branch points $f: \mathbb{S}^{2} \longrightarrow \mathbb{S}^{2 m}$ of harmonic degree $d$. Since it is maximal for the first eigenvalue, we have $N(2)=1$. It follows from Theorem 4.5 that if $d>1$ then $1 \geqslant d+1$, which is impossible. Therefore, $d=1$, and hence $m=1$ by Proposition 4.4. Moreover, Area $\left(\mathbb{S}^{2}, f^{*} g_{\mathbb{S}^{2}}\right)=4 \pi$, which implies that $f$ is a one-sheeted covering. In particular, it does not have branch points, and thus a maximal metric is smooth.

We now prove (3) by induction in $k$. Indeed, the result holds for $k=1$ as was mentioned above. Suppose that it holds for $j=1, \ldots, k-1$. Assume, by contradiction, that $\Lambda_{k}\left(\mathbb{S}^{2}\right)>8 \pi k$. Then by Theorem 2.3 there exists a maximizing metric $g$ which is smooth possibly with the exception of a finite number of conical singularities such that $\Lambda_{k}\left(\mathbb{S}^{2}\right)=\bar{\lambda}_{k}\left(\mathbb{S}^{2}, g\right)$. By Proposition 4.4 the metric $g$ is induced on $\mathbb{S}^{2}$ by a linearly full harmonic immersion (possibly with branch points) $f: \mathbb{S}^{2} \longrightarrow \mathbb{S}^{2 m}$ of harmonic degree $d \geqslant 1$. Note that by Theorem 3.9 ,

$$
\lambda_{k}\left(\mathbb{S}^{2}, g\right)=2 .
$$

At the same time,

$$
\lambda_{k}\left(\mathbb{S}^{2}, g\right)=2>\lambda_{j}\left(\mathbb{S}^{2}, g\right)
$$

for all $j=0,1, \ldots, k-1$, and therefore

$$
N(2)=k \text {. }
$$


Indeed, it follows from the induction hypothesis that for any $j<k$,

$\lambda_{k}\left(\mathbb{S}^{2}, g\right)$ Area $\left(\mathbb{S}^{2}, g\right)=\Lambda_{k}\left(\mathbb{S}^{2}\right)>8 \pi k>8 \pi j=\Lambda_{j}\left(\mathbb{S}^{2}\right) \geqslant \lambda_{j}\left(\mathbb{S}^{2}, g\right)$ Area $\left(\mathbb{S}^{2}, g\right)$,

which immediately implies inequality (10).

Consider the case $d>1$. Then Theorem 4.5 implies that

$$
N(2)=k \geqslant d+1 \text {. }
$$

On the other hand, by Definition 4.3 we have

$$
\text { Area }\left(\mathbb{S}^{2}, g\right)=4 \pi d \text {. }
$$

Therefore, combining (91) and (11) we get

$$
\Lambda_{k}\left(\mathbb{S}^{2}\right)=\bar{\lambda}_{k}\left(\mathbb{S}^{2}, g\right)=2 \operatorname{Area}\left(\mathbb{S}^{2}, g\right)=8 \pi d<8 \pi k
$$

which contradicts the assumption $\Lambda_{k}\left(\mathbb{S}^{2}\right)>8 \pi k$.

Finally, consider the case $d=1$. Then Area $\left(\mathbb{S}^{2}, g\right)=4 \pi$ and

$$
\bar{\lambda}_{k}\left(\mathbb{S}^{2}, g\right)=8 \pi \text {. }
$$

However, in this case the metric $g$ could be maximal only for $k=1$, but we have assumed that $k>1$. Therefore $\Lambda_{k}\left(\mathbb{S}^{2}\right)=8 \pi k$, for any $k \geqslant 2$, and it follows from Remark 2.4 that the equality is attained in the limit by a sequence of metrics degenerating to a union of $k$ touching identical round spheres. This completes the proof of the theorem.

\section{Appendix A. Theorem 4.5: outline of the Proof}

The goal of this Appendix is to present the main ideas of the proof of Theorem 4.5 of 11 which is a key ingredient in the proof of Theorem 1.2, The techniques used in [11] are not well-known in the spectral geometry community, but they appear to be quite powerful (see [46] for another recent application), and we believe it is worth discussing them in some detail. In particular, for the convenience of the reader, we recall a number of notions from algebraic geometry that are used in the proof of Theorem 4.5.

I. Isotropy. Recall that there exists a unique complex structure on the sphere $\mathbb{S}^{2}$. Let $z$ be a local complex coordinate, and denote by $\partial$ and $\bar{\partial}$ derivatives with respect to $z$ and $\bar{z}$ respectively. One can easily check that the definitions below do not depend on the choice of $z$. For two vectors $u, v \in \mathbb{C}^{n}$ we use $(u, v)=\sum_{i=1}^{n} u_{i} v_{i}$ to denote $\mathbb{C}$-bilinear inner product and use $\langle u, v\rangle=(u, \bar{v})=$ $\sum_{i=1}^{n} u_{i} \bar{v}_{i}$ to denote the Hermitian inner product. The symbol $\perp$ refers to orthogonality in the Hermitian metric.

Definition A.1. A subspace $V$ of $\mathbb{C}^{n}$ is called isotropic if $\left(v_{1}, v_{2}\right)=0$ for all $v_{1}, v_{2} \in V$. Equivalently, $V$ is isotropic if $V \perp \bar{V}$ with respect to the Hermitian inner product on $\mathbb{C}^{n}$. 
Definition A.2. A branched conformal immersion $x: \mathbb{S}^{2} \rightarrow \mathbb{S}^{n} \subset \mathbb{R}^{n+1}$ is called isotropic if for all $k+l \geqslant 1$ one has $\left(\partial^{k} x, \partial^{l} x\right)=0$, i.e. for any point $p \in \mathbb{S}^{2}$, the complex derivatives of $x$ at $p$ span an isotropic subspace in $\mathbb{C}^{n+1}$.

Calabi [6] proved the following theorem.

Theorem A.3. Any linearly full branched minimal immersion $x: \mathbb{S}^{2} \rightarrow \mathbb{S}^{n}$ is isotropic.

Remark A.4. Note that in the literature, isotropic minimal immersions to $\mathbb{S}^{4}$ are sometimes referred to as superminimal. The notion of superminimal immersion was introduced in the paper of Bryant [5] though the definition there differs from Definition A.2

As a corollary one obtains the statement (ii) of Theorem 4.2, i.e. $n=2 m$.

Proof. Fix a point $p \in \mathbb{S}^{2}$. Let $G$ be an isotropic subspace spanned by complex derivatives of $x$ at $p$. Then $G$ is isotropic, i.e. $G \perp \bar{G}$. Moreover, as $x$ sends $\mathbb{S}^{2}$ to the unit sphere, one has $(x, x)=1$. Applying $\partial^{k}$ and $\bar{\partial}^{k}$ to this relation and using the isotropy of $G$ and $\bar{G}$ one obtains $x(p) \perp G$ and $x(p) \perp \bar{G}$.

Suppose that there exists a constant vector $u \in \mathbb{R}^{n+1}$ such that $u \perp$ $\mathbb{C} x(p) \oplus G \oplus \bar{G}$. Then $(x, u)$ has a zero of infinite order at $p$ and by the unique continuation theorem $(x, u) \equiv 0$, which contradicts the fact that $x$ is linearly full. Therefore, $\mathbb{C}^{n+1}=\mathbb{C} x \oplus G \oplus \bar{G}$, i.e. $n=2 m$.

II. Directrix curve. To any linearly full harmonic map $x: \mathbb{S}^{2} \rightarrow \mathbb{S}^{2 m}$, one can canonically associate a holomorphic curve $\Phi: \mathbb{S}^{2} \rightarrow \mathbb{C P}^{2 m}$ called the directrix curve. In order to introduce it we need the two auxiliary definitions below.

Definition A.5. For a holomorphic curve $\Psi: \mathbb{S}^{2} \rightarrow \mathbb{C P}^{n}$, the $k$-th associated curve $\Psi_{k}$ is the holomorphic map $\Psi_{k}: \mathbb{S}^{2} \rightarrow G r_{k+1, n+1}(\mathbb{C})$ to a complex Grassmannian, which in terms of any local holomorphic lift $\psi: U \rightarrow \mathbb{C}^{n+1}$ is given by a $k$-subspace spanned by $\psi, \partial \psi, \ldots, \partial^{k} \psi$.

Definition A.6. A linearly full holomorphic curve $\Psi: \mathbb{S}^{2} \rightarrow \mathbb{C P}^{2 m}$ is called isotropic if its $(m-1)$-th associated curve takes values in isotropic $m$-planes in $\mathbb{C}^{2 m+1}$.

For each isotropic curve $\Psi: \mathbb{S}^{2} \rightarrow \mathbb{C P}^{2 m}$, one can construct a pair of linearly full harmonic maps $\pm x x_{\Psi} \mathbb{S}^{2} \rightarrow \mathbb{S}^{2 m}$ in the following way. In view of isotropy, $\Psi_{m-1} \perp \bar{\Psi}_{m-1}$ as $m$-dimensional subspaces of $\mathbb{C}^{2 m+1}$. Thus, $\Psi_{m-1} \oplus \bar{\Psi}_{m-1}$ spans a $2 m$-dimensional subspace, which is invariant under conjugation. Therefore, its orthogonal complement is also invariant under conjugation, and we define $\tilde{x}_{\Psi}: \mathbb{S}^{2} \rightarrow \mathbb{R P}^{2 m}$ as a unique real line in $\left(\Psi_{m-1} \oplus \bar{\Psi}_{m-1}\right)^{\perp}$. Barbosa proved [3] that such $\tilde{x}_{\Psi}$ is harmonic. As $\mathbb{S}^{2}$ is simply connected, $\tilde{x}_{\Psi}$ can be lifted to a pair of linearly full harmonic maps $\pm x_{\Psi}: \mathbb{S}^{2} \rightarrow \mathbb{S}^{2 m}$.

Proposition A.7 (Barbosa [3]). For any linearly full harmonic map $x: \mathbb{S}^{2} \rightarrow$ $\mathbb{S}^{2 m}$ there exists unique isotropic curve $\Phi: \mathbb{S}^{2} \rightarrow \mathbb{C P}^{2 m}$ such that $\pm x_{\Phi}=x$. 
The curve $\Phi$ is called directrix curve of the linearly full harmonic map $x$. In what follows we do not distinguish between $x$ and $-x$, see Remark A.10.

Idea of the proof. For each $i=1, \ldots, m$, define $G_{i}$ inductively as follows: $G_{1}=\bar{\partial} x$ and

$$
G_{i}=\bar{\partial}^{i} x+\sum_{j=1}^{i-1} a_{i}^{j} G_{j},
$$

where the coefficients $a_{i}^{j}$ are chosen in such a way that $\left\langle G_{i}, G_{j}\right\rangle=0$ for $i \neq j$. Then $\Phi=\pi \circ G_{m}$, where $\pi: \mathbb{C}^{2 m+1} \backslash\{0\} \rightarrow \mathbb{C P}^{2 m}$ is the natural projection.

III. Harmonic degree. In this section we give another interpretation of the harmonic degree of an isotropic branched minimal immersion, see Definition 4.3 .

Let $\xi_{k, n}: G r_{k, n}(\mathbb{C}) \rightarrow \mathbb{C P}\left(\begin{array}{l}n \\ k\end{array}\right)-1$ denote the Plücker embedding, see e.g. [20]. Let $\tilde{d}$ be the algebraic degree of the curve

$$
\xi_{m, 2 m+1} \circ \Phi_{m-1}: \mathbb{S}^{2} \rightarrow \mathbb{C P}\left(\begin{array}{c}
2 m+1 \\
m
\end{array}\right)-1 .
$$

Then one has the following theorem.

Theorem A.8 (Barbosa [3]). The degree $\tilde{d}$ is even. Moreover, it is equal to twice the harmonic degree $d$.

$I V$. Action of $S O(2 m+1, \mathbb{C})$. Barbosa 3 noticed that the group $S O(2 m+$ $1, \mathbb{C})$ naturally acts on the space of linearly full isotropic branched minimal immersions. Indeed, let $A \in S O(2 m+1, \mathbb{C})$ and let $\Phi$ be the directrix curve of $x: \mathbb{S}^{2} \rightarrow \mathbb{S}^{2 m}$ (see subsection II). Then $A \Phi$ is an isotropic curve in $\mathbb{C P}^{2 m}$, and therefore it is the directrix curve of some linearly full isotropic branched minimal immersion that we denote by $A x$.

In the same way, $S O(2 m+1, \mathbb{C})$ acts on $m$-dimensional subspaces, and it is easy to see that $(A \Phi)_{m-1}=A\left(\Phi_{m-1}\right)$. Thus, this action preserves the harmonic degree of the immersion $x$. This fact will be used in Proposition A.12.

$V$. Conformally covariant Schrödinger operators. To each conformal map $x: \mathbb{S}^{2} \rightarrow \mathbb{S}^{2 m}$ one can associate 38] a conformally covariant Schrödinger operator $L_{x}$ in the following way. For any metric $g$ on $\mathbb{S}^{2}$ we set

$$
L_{x, g}=\Delta_{g}-|\nabla x|_{g}^{2},
$$

where $|\nabla x|_{g}^{2}=\sum_{i=1}^{2 m+1}\left|\nabla x_{i}\right|_{g}^{2}$. It is easy to see that for any function $\omega \in$ $C^{\infty}\left(\mathbb{S}^{2}\right)$ one has $L_{x, e^{2 \omega} g}=e^{-2 \omega} L_{x, g}$. Thus, the corresponding quadratic form

$$
Q_{x}(u)=\int_{\mathbb{S}^{2}}|\nabla u|_{g}^{2}-|\nabla x|_{g}^{2} u^{2} d v_{g}
$$

is conformally invariant. This fact motivates the following definition. 
Definition A.9. The index $\operatorname{Ind}(x)$ of a conformal map $x: \mathbb{S}^{2} \rightarrow \mathbb{S}^{2 m}$ is defined to be the index of $L_{x, g}$, i.e. the number of negative eigenvalues of $L_{x, g}$.

Similarly, the nullity $\operatorname{Nul}(x)$ of a conformal map $x: \mathbb{S}^{2} \rightarrow \mathbb{S}^{2 m}$ is defined as the nullity of $L_{x, g}$, i.e. dim $\operatorname{ker} L_{x, g}$. Both index and nullity do not depend on the choice of the metric $g$.

Remark A.10. Clearly, $\operatorname{Ind}(x)=\operatorname{Ind}(-x)$ and $\operatorname{Nul}(x)=\operatorname{Nul}(-x)$.

Remark A.11. An elementary computation shows that for $g=x^{*} g_{\mathbb{S}^{2 m}}$ the operator $L_{x, g}$ takes the form

$$
L_{x, g}=\Delta_{g}-2
$$

i.e. $\operatorname{Ind}(x)$ coincides with the number $N(2)$ appearing in the statement of Theorem 4.5 .

VI. Proof of Theorem 4.5. The following proposition summarizes some of the results of the paper [11].

Proposition A.12 (Ejiri [11]). Let $x: \mathbb{S}^{2} \rightarrow \mathbb{S}^{2 m}, m>1$, be a linearly full isotropic branched minimal immersion of harmonic degree $d>1$. Then there exists a one-parameter subgroup $A(t) \subset S O(2 m+1, \mathbb{C})$ such that

1) Ast $\rightarrow \infty$, the one-parameter family $x_{t}=A(t) x$ converges uniformly to a linearly full isotropic branched minimal immersion $x_{\infty}: \mathbb{S}^{2} \rightarrow$ $\mathbb{S}^{2 m-2}$ of harmonic degree $d$, where $\mathbb{S}^{2 m-2} \subset \mathbb{S}^{2 m}$ is an equatorial sphere;

2) $\operatorname{Nul}(x)=\operatorname{Nul}\left(x_{t}\right) \leqslant \operatorname{Nul}\left(x_{\infty}\right)$;

3) $\operatorname{Ind}(x)=\operatorname{Ind}\left(x_{t}\right) \geqslant \operatorname{Ind}\left(x_{\infty}\right)$.

In the first statement we are using the degree-preserving property of the action of $S O(2 m+1, \mathbb{C})$ which was explained in subsection IV. Note also that the third statement follows from the second one by continuity of eigenvalues of the Schrödinger operator $L_{x, g}$ with respect to continuous deformations of the potential.

If $m>2$, one can apply the previous proposition to $x_{\infty}$ and obtain a new isotropic branched minimal immersion to $\mathbb{S}^{2 m-4}$. Continuing this process, we arrive at a linearly full isotropic branched minimal immersion $y: \mathbb{S}^{2} \rightarrow \mathbb{S}^{2}$ of harmonic degree $d$. Moreover, $\operatorname{Ind}(x) \geqslant \operatorname{Ind}(y)$. At the same time, the following result holds:

Theorem A.13 (Montiel and Ros [38, Theorem 21]; Nayatani [45]). Let $y: \mathbb{S}^{2} \rightarrow \mathbb{S}^{2}$ be a conformal map of degree $d>1$. Then $\operatorname{Ind}(y) \geqslant d+1$.

Therefore, given a linearly full harmonic map $x: \mathbb{S}^{2} \rightarrow \mathbb{S}^{2 m}$ of degree $d>1$, in view of Remark A.11 we have $N(2)=\operatorname{Ind}(x) \geqslant \operatorname{Ind}(y) \geqslant d+1$, which completes the proof of Theorem 4.5. 


\section{REFERENCES}

[1] P. Antunes, P. Freitas, Numerical optimization of low eigenvalues of the Dirichlet and Neumann Laplacians, J. Optim. Theory Appl., 154:1 (2012), 235-257.

[2] S. Bando, H. Urakawa, Generic properties of the eigenvalue of Laplacian for compact Riemannian manifolds. Tôhoku Math. J., 35:2 (1983), 155-172.

[3] J. Barbosa, On minimal immersions of $S^{2}$ into $S^{2 m}$, Trans. Amer. Math. Soc., 210 (1975), 75-106.

[4] M. Berger, Sur les premières valeurs propres des variétés Riemanniennes. Compositio Math., 26 (1973), 129-149.

[5] R. L. Bryant, Conformal and minimal immersions of compact surfaces into the 4sphere, J. Differential Geom. 17:3 (1982), 455-473.

[6] E. Calabi, Minimal immersions of surfaces in Euclidean spheres, J. Differential Geometry, 1 (1967), 111-125.

[7] J. Eells, L. Lemaire, A report on harmonic maps, Bull. London Math. Soc., 10:1 (1978), 1-68.

[8] D. Cianci, M. Karpukhin, V. Medvedev, On branched minimal immersions of surfaces by first eigenfunctions. Preprint arXiv:1711.05916

[9] B. Colbois, A. El Soufi, Extremal eigenvalues of the Laplacian in a conformal class of metrics: the conformal spectrum, Ann. Global Anal. Geom., 24:4 (2003), 337-49.

[10] J. Eells, J. H. Sampson, Harmonic mappings of Riemannian manifolds, Amer. J. Math., 86 (1964), 109-160.

[11] N. Ejiri, The boundary of the space of full harmonic maps of $\mathbf{S}^{2}$ into $\mathbf{S}^{2 m}(1)$ and extra eigenfunctions, Japan. J. Math, 24:1 (1998), 83-121.

[12] N. Ejiri, M. Kotani, Minimal Surfaces in $\mathbb{S}^{2 m}(1)$ with extra eigenfunctions, The Quarterly Journal of Mathematics, 43:4 (1992), 421-440.

[13] A. El Soufi, H. Giacomini, M. Jazar, A unique extremal metric for the least eigenvalue of the Laplacian on the Klein bottle, Duke Math. J., 135:1 (2006), 181-202. Preprint arXiv: math/0701773.

[14] A. Girouard, N. Nadirashvili, I. Polterovich, Maximization of the second positive Neumann eigenvalue for planar domains, J. Diff. Geom., 83 (2009), 637-661. Preprint arXiv:0801.2142.

[15] A. Girouard, I. Polterovich, On the Hersch-Payne-Schiffer estimates for the eigenvalues of the Steklov problem, Funktsional. Anal. i Prilozhen., 44:2 (2010), 33-47 (Russian), translated in Funct. Anal. Appl., 44:2 (2010), 106-117.

[16] A. El Soufi, S. Ilias, Le volume conforme et ses applications d'après Li et Yau, Sém. Théorie Spectrale et Géométrie, Institut Fourier, 1983-1984, No.VII, (1984).

[17] A. El Soufi, S. Ilias, Immersions minimales, première valeur propre du laplacien et volume conforme, Math. Ann., 275:2 (1986), 257-267.

[18] A. El Soufi, S. Ilias, Riemannian manifolds admitting isometric immersions by their first eigenfunctions, Pacific J. Math., 195:1 (2000), 91-99.

[19] A. El Soufi, S. Ilias, Laplacian eigenvalue functionals and metric deformations on compact manifolds, J. Geom. Phys., 58:1 (2008), 89-104. Preprint arXiv:math/0701777.

[20] P. Griffiths, J. Harris, Principles of algebraic geometry. John Wiley \& Sons, 1978.

[21] R. D. Gulliver II, R. Osserman, H. L. Royden, A Theory of Branched Immersions of Surfaces, Amer. J. Math., 95:4 (1973), 750-812.

[22] A. Hassannezhad, Conformal upper bounds for the eigenvalues of the Laplacian and Steklov problem, J. Funct. Anal. 261 (2011), 3419-3436.

[23] J. Hersch, Quatre propriétés isopérimétriques de membranes sphériques homogènes, C. R. Acad. Sci. Paris Sér A-B, 270 (1970), A1645-A1648.

[24] D. Jakobson, M. Levitin, N. Nadirashvili, N. Nigam, I. Polterovich, How large can the first eigenvalue be on a surface of genus two? Int. Math. Res. Not. 63 (2005), 3967-3985. Preprint arXiv:math/0509398. 
[25] D. Jakobson, N. Nadirashvili, I. Polterovich, Extremal Metric for the First Eigenvalue on a Klein Bottle, Canad. J. Math., 58:2 (2006), 381-400. Preprint arXiv:math/0311484.

[26] C.-Y. Kao, R. Lai, B. Osting, Maximization of Laplace-Beltrami eigenvalues on closed Riemannian surfaces, ESAIM Control Optim. Calc. Var., 23:2 (2017), 685720. Preprint arXiv: 1405.4944.

[27] M. A. Karpukhin, Nonmaximality of extremal metrics on a torus and the Klein bottle (in Russian), Mat. Sb., 204:12 (2013), 31-48; English translation in Sb. Math., 204:11-12 (2013), 1728-1744. Preprint arxiv:1210.8122.

[28] M. A. Karpukhin, Spectral properties of bipolar surfaces to Otsuki tori, J. Spectr. Theory, 4:1 (2014), 87-111. Preprint arXiv:1205.6316.

[29] M. Karpukhin, Spectral properties of a family of minimal tori of revolution in five-dimensional sphere, Canad. Math. Bull., 58:2 (2015), 285-296. Preprint arXiv: 1301.2483.

[30] M. Karpukhin, Upper bounds for the first eigenvalue of the Laplacian on nonorientable surfaces, Int. Math. Res. Notices, 2016:20 (2016), 6200-6209. Preprint arXiv: 1503.08493.

[31] G. Kokarev, Variational aspects of Laplace eigenvalues on Riemannian surfaces, Adv. Math. 258 (2014), 191-239. Preprint arXiv:1103.2448.

[32] G. Kokarev, Conformal volume and eigenvalue problems. Preprint arXiv: 1712.08150 .

[33] N. Korevaar, Upper bounds for eigenvalues of conformal metrics, J. Differential Geom., 37:1 (1993), 73-93.

[34] H. Lapointe, Spectral properties of bipolar minimal surfaces in $\mathbb{S}^{4}$, Differential Geom. Appl., 26:1 (2008), 9-22. Preprint arXiv:math/0511443.

[35] P. Li, S.-T. Yau, A new conformal invariant and its applications to the Willmore conjecture and the first eigenvalue of compact surfaces, Invent. Math., 69:2 (1982), 269-291.

[36] P. Li, S.-T. Yau, On the Schrodinger equation and the eigenvalue problem, Comm. Math. Phys, 88:3 (1983), 309-318.

[37] S. Montiel, A. Ros, Minimal immersions of surfaces by the first eigenfunctions and conformal area, Invent. Math., 83 (1986), 153-166.

[38] S. Montiel, A. Ros, Schrödinger operators associated to a holomorphic map, Global Differential Geometry and Global Analysis, 147-174, Lecture Notes in Math., 1481, Springer, Berlin, 1991.

[39] N. Nadirashvili, Berger's isoperimetric problem and minimal immersions of surfaces. Geom. Funct. Anal., 6:5 (1996), 877-897.

[40] N. Nadirashvili, Isoperimetric inequality for the second eigenvalue of a sphere, J. Differential Geom., 61:2 (2002), 335-340.

[41] N. S. Nadirashvili, A. V. Penskoi, An isoperimetric inequality for the second non-zero eigenvalue of the Laplacian on the projective plane, Geom. Funct. Anal., 28:5 (2018), 1368-1393. Preprint arXiv: 1608.07334.

[42] N. Nadirashvili, Y. Sire, Conformal spectrum and harmonic maps, Mosc. Math. J., 15:1 (2015), 123-140, 182. Preprint arXiv:1007.3104.

[43] N. Nadirashvili, Y. Sire, Maximization of higher order eigenvalues and applications, Mosc. Math. J., 15:4 (2015), 767-775. Preprint arXiv:1504.07465.

[44] N. Nadirashvili, Y. Sire, Isoperimetric inequality for the third eigenvalue of the Laplace-Beltrami operator on $\mathbb{S}^{2}$, J. Differential Geometry, 107:3 (2017), 561-571. Preprint arXiv:1506.07017.

[45] S. Nayatani, Morse index and Gauss maps of complete minimal surfaces in Euclidean 3-space, Comm. Math. Helv. 68:1 (1993) 511-537. 
[46] S. Nayatani, T. Shoda, Metrics on a closed surface of genus two which maximize the first eigenvalue of the Laplacian, C. R. Math. Acad. Sci. Paris 357:1 (2019), 84-98. Preprint arXiv: 1704.06384.

[47] A. V. Penskoi, Extremal spectral properties of Lawson tau-surfaces and the Lamé equation, Moscow Math. J. 12:1 (2012), 173-192. Preprint arXiv:1009.0285.

[48] A. V. Penskoi, Extremal spectral properties of Otsuki tori, Math. Nachr., 286:4 (2013), 379-391. Preprint arXiv:1108.5160.

[49] A. V. Penskoi, Extremal metrics for the eigenvalues of the Laplace-Beltrami operator on surfaces (in Russian), Uspekhi Mat. Nauk, 68:6(414) (2013), 107-168; English translation in Russian Math. Surveys, 68:6 (2013), 1073-1130.

[50] A. V. Penskoi, Generalized Lawson tori and Klein bottles, J. Geom. Anal., 25:4 (2015), 2645-2666. Preprint arXiv:1308.1628.

[51] R. Petrides, Existence and regularity of maximal metrics for the first Laplace eigenvalue on surfaces, Geom. Funct. Anal. 24:4 (2014), 1336-1376.

[52] R. Petrides, Maximization of the second conformal eigenvalue of spheres, Proc. Amer. Math. Soc., 142:7 (2014), 2385-2394. Preprint arXiv:1206.0229.

[53] R. Petrides, On the existence of metrics which maximize Laplace eigenvalues on surfaces, Internat. Math. Res. Not., 2017, pp. 1-96.

[54] G. Pólya, Patterns of plausible inference. Mathematics and plausible reasoning, Vol. II. Princeton Univ. Press, Princeton, N.J., 1954. x+190 pp.

[55] G.Pólya, On the eigenvalues of vibrating membranes, Proc. London Math. Soc. (3) 11 (1961), 419-433.

[56] R. Schoen, S.-T. Yau, Lectures on differential geometry. Conference Proceedings and Lecture Notes in Geometry and Topology, I. International Press, Cambridge, MA, 1994.

[57] D. A. Sher, Conic degeneration and the determinant of the Laplacian, J. Anal. Math., 126 (2015), 175-226. Preprint arXiv:1208.1809.

[58] T. Takahashi, Minimal immersions of Riemannian manifolds, J. Math. Soc. Japan, 18:4 (1966), 380-385.

[59] P. C. Yang, S.-T. Yau, Eigenvalues of the Laplacian of compact Riemann surfaces and minimal submanifolds, Ann. Scuola Norm. Sup. Pisa Cl. Sci., 7:1 (1980), 55-63. 
(Mikhail Karpukhin) Department of Mathematics and Statistics, McGill University, Burnside Hall, 805 Sherbrooke Street West, Montréal, Québec, H3A 0B9, CANADA

Current address: Department of Mathematics, University of California, Irvine, CA 92697, USA

E-mail address: mkarpukh@uci.edu

(Nikolai Nadirashvili) CNRS, I2M UMR 7353 - Centre de Mathématiques et Informatique, Marseille, France

E-mail address: nikolay.nadirashvili@univ-amu.fr

(Alexei V. Penskoi) Department of Higher Geometry and Topology, Faculty of Mathematics and Mechanics, Moscow State University, Leninskie Gory, GSP-1, 119991, Moscow, Russia

and

Faculty of Mathematics, National Research University Higher School of Economics, 6 Usacheva Str., 119048, Moscow, Russia

and

Interdisciplinary Scientific Center J.-V. Poncelet (ISCP, UMi 2615), Bolshoy Vlasyevskiy Pereulok 11, 119002, Moscow, Russia

and

(Visiting position) CNRS, I2M UMR 7353 - Centre de Mathématiques et Informatique, Marseille, France

E-mail address, Corresponding author: penskoi@mccme.ru

(Iosif Polterovich) Département de mathématiques et de Statistique, Université de Montréal, CP 6128 Succ. Centre-Ville, Montréal, Québec, H3C 3J7, CANADA

E-mail address: iossif@dms.umontreal.ca 